\title{
Editorial Introduction: Proceedings of the Second Satellite Symposium on Complex Systems and Education, Held at the Conference on Complex Systems, Cancun, Mexico, September 20, 2017
}

\author{
MATTHIJS KOOPMANS \\ Mercy College (USA) \\ HIROKI SAYAMA \\ Binghamton University (USA)
}

Welcome to the proceedings of the Second CCS Satellite Symposium on Complex Systems and Education, which was held in the course of the Conference on Complex Systems (CCS) in Cancun, Mexico, in late September of 2017. CCS is an interdisciplinary forum for scholars who share an interest in complexity theory and other dynamical perspectives. The discussion of complexity as an educational phenomenon is a relatively new development in the complexity field that has yielded some significant empirical results as well as interesting and important insights about the use of complexity theory for teaching and learning.

As complex systems science has matured as an interdisciplinary field, its paradigms and essential concepts, such as dynamical systems, sensitivity in initial conditions, chaos, stochasticity, interdependence, self-organization, phase transition, learning, evolution, networks, multiscale properties and emergence, have been widely adopted in a number of scientific disciplines, from biology to social sciences and from engineering to medicine. To this date, educational research has remained heavily reliant on conventional paradigms, which permits only a limited range of questions about education to be investigated. Meanwhile, work on complexity in education has been largely theoretical and exploratory, without having the level of conceptual and methodological specificity that is required to capture the dynamical processes hypothesized in the complex systems literature, nor does it speak to the specific gaps in our knowledge that result from the relative absence of dynamical perspectives in empirical educational research. Likewise, the key concepts of complex systems have remained mostly 
excluded from science as taught in the K-16 education setting. Recent progress in complex systems science includes significant and path-breaking empirical work to study the dynamical underpinnings of the educational process, and substantial development of structured, accessible educational materials about complexity.

This satellite symposium presented some of the advances at the intersection of educational research and complex systems science, and thereby it promoted cross-fertilization of ideas among researchers and growth of this important area of research and practice. Of the eight presentations that were given at the satellite (https://mkoopmans.wixsite.com/ccs-2017-edsymposium), four papers are included in these proceedings, in which two presentations by Gwen Marchand (University of Nevada) and Jonathan Hilpert (Georgia State University) were condensed by the authors into a single paper. The resulting three contributions represent the two major areas of application that was the focus of the satellite, namely (1) the implications of complexity theory for educational research and (2) the use of complexity theory for curriculum and instruction at kindergarten and the elementary and secondary school levels.

The first contribution by Towlson et al. is pragmatic in its focus, as it utilizes network science as a professional development tool for K-12 teachers. Twenty-one teachers of varying disciplinary orientations and grade levels participated in a workshop, which included a section in which network concepts were discussed and a section focusing on lesson planning, and the mapping of standards to curricular modules using network science. The paper shares information about the lesson plans that were developed, as well participants' evaluations about what they learned at the workshop. This paper illustrates the relevance of complexity theory to the enhancement of educational practice.

An entirely different angle to the use of complexity theory is presented by Koopmans and by Marchand and Hilpert, whose focus is on its use to further enhance educational research methods, and address questions that would not appear on our radar were it not for the concerns and priorities of the theory. Koopmans' study concerns the daily attendance rates in an urban transfer high school that made a transition from being a large high school with an enrollment of close to one thousand students to a smaller school with around 250 students. With respect to daily attendance, conventional linear views would inspire one to determine whether the average daily attendance were higher after than before the reduction in school size, while complexity theory would inspire one to ask one critical additional question which is whether the reduction in size of the school has a stabilizing effect on attendance. The paper provides the methodological details about how this question can be addressed statistically. Marchand and Hilpert present a necessary and broader discussion about how our priorities are shifting in educational research once a complexity angle is used, and they present an overview of the main design features of a complexity approach, to wit, a focus on context in which systems function and on the time scale over which adaptive behavior plays out. The paper provides examples of existing complexity research in education, and how it represents these two perspectives.

These proceedings attest to the vigor of the field of educational complexity, and its use for purposes of instruction and professional development as well as the development of more sophisticated research designs that will allow researchers to penetrate more deeply into the

Complicity: An International Journal of Complexity and Education Volume 15 (2018), Number 1・pp. 1-3 
dynamical underpinnings of the systemic aspects of the educational process. The insight we gain along the way can in turn be used to provide more effective feedback to practitioners and policy makers in the field and hopefully further support our attempts to address some of the major challenges with which the field is beset, such as the gap in student achievement between demographic groups, the inertia of the P-12 educational system and the need for better support for those working in the field. In addition, there is an ongoing need in the field of complexity theory for prototypical examples of complex processes, of which there are many in education, as the papers presented here vividly illustrate.

\section{About the Authors}

Matthijs Koopmans is a Professor in the Department of Educational Leadership at the School of Education, Mercy College. His scholarly interests include the applicability of nonlinear dynamical systems to educational organizations and school reform, cause and effect relationships and long memory processes in time series data. Correspondence: $\underline{\text { mkoopmans@mercy.edu }}$

Hiroki Sayama is a Professor in the Department of Systems Science and Industrial Engineering, and the Director of the Center for Collective Dynamics of Complex Systems (CoCo), at Binghamton University, State University of New York. His expertise and interests include complex dynamical networks, human and social dynamics, collective behaviors, artificial life/chemistry, interactive systems, and complex systems education, among others.

Correspondence: sayama@binghamton.edu

Complicity: An International Journal of Complexity and

Education Volume 15 (2018), Number 1・pp. 1-3 